\title{
Physiological Characteristics and Nonparametric Test for Master-Slave Driving Task's Mental Workload Evaluation in Mountain Area Highway at Night
}

\author{
Haiwei Wang, ${ }^{1}$ Jianrong Liu, ${ }^{2}$ and Feng You $\mathbb{D}^{2}$ \\ ${ }^{1}$ School of Transportation and Economic Management, Guangdong Communication Polytechnic, Guangzhou 510650, China \\ ${ }^{2}$ School of Civil Engineering and Transportation, South China University of Technology, Guangzhou 510640, China \\ Correspondence should be addressed to Feng You; youfeng77@126.com
}

Received 25 August 2019; Accepted 15 October 2019; Published 3 December 2019

Guest Editor: Feng Chen

Copyright (C) 2019 Haiwei Wang et al. This is an open access article distributed under the Creative Commons Attribution License, which permits unrestricted use, distribution, and reproduction in any medium, provided the original work is properly cited.

\begin{abstract}
With the rapid development of advanced mobile intelligent terminals, driving tasks are diverse, and new traffic safety problems occur. We propose a new research on physiological characteristics and nonparametric tests for the master-slave driving task, especially for evaluation of drivers' mental workload in mountain area highway in nighttime scenario. First, we establish the experimental platform based driving simulator and design the master-slave driving task. Second, based on the physiological data and subjective evaluation for mental workload, we use statistical methods to composite the physical changes evolution analysis in a driving simulator. Finally, we finished nonparametric test of the drivers' psychological load and road test. The results show that in compassion with the daytime scenario, drivers should pay much effort to driving skills and risk identification in the nighttime scenario. Thus, in the same driving condition, drivers should bear the higher level of mental workload, and it has been subjected to even greater pressures and intensity of emotions.
\end{abstract}

\section{Introduction}

1.1. Problem and Motivation. Driving safety is one of the eternal themes in road traffic engineering. When the advanced driver assistance system (ADAS) and on-board intelligent terminals have been used increasingly, the drivers' mental workload changed from the single task to multiple tasks. The drivers cannot obtain road-related information due to excessive mental stress, which will influence decision-making for driving. And the drivers are exposed to the frequent traffic accidents.

In the field of cognitive psychology, human cognitive resources are limited. They have to allocate the resources to spatiotemporal scenarios and activities when engaged in a psychological activity, namely, the drivers will expend more cognitive resources if they are involved in multiple driving tasks. Accordingly, for the driving behavior, drivers cannot quickly collect and process environmental and driving information. The risk of road traffic accidents increases.

1.2. Related Works. In traffic accident research, the driver's own reasons cause cognitive errors in the road traffic environment, the traffic state is judged incorrectly. And the proportion on traffic accidents is caused by slow brain response accounting for more than half. Especially, relevant traffic safety research shows that the number on traffic accidents caused by the driver's own responsibility, especially for traffic fatal accidents, is increasing year by year. In response to this problem, researchers have carried out research working on the mental load on the driver during driving. Some researchers used FMRI and FNIRS to collect data on the driver's brain physiological activity. Through driving experiments, the researchers selected significant physiological indicators for evaluating brain mental load [1-4]. Based on the physiological indicators and Human Factors Engineering, studies have considered changes in heart rate variability power spectral density. In the driving task, the driver's mental load increases as driving tasks increase, but the heart rate variability power spectral density decreases [5-7].

With the application and the promotion of physiological sensors, researchers have applied physiological instruments to collect the driver's electrocardiogram and EEG data $[8,9]$. Based on the above data, a driving mental load prediction model is established $[10,11]$ and the rationality of human-computer interaction analyzed [12]. 
Some researchers use driving simulators to analyze the relationship between the driver's mental load and driver's vision [13]. In addition, based on real driving scenarios, the research has focused on the impact of using mobile phones on driving behavior $[14,15]$ and road safety [16].

As the research progressed, the researchers designed driving experiments on simulators to analyze the gaze point variation for the driver's view field under different mental loads [17]. There is a significant difference in the distribution of the gaze points under mental load levels. Specifically, as the mental load increases, the concentrated area of the gaze points will shrink [18].

In references [19-21], the driver's psychological load also has a greater impact on travel time, especially at intersections [22-24]. For semiautonomous vehicles [25], the humanmachine sharing technology allows the driver and the OBU to share control of the vehicle, reducing the driver's psychological load [26-28]. In addition, intelligence, networking, and electrification have become the trend of the current automotive industry. In response to the demand for new energy vehicles bench testing [29], part of the research is devoted to the relationship between human-machine interface design and driver's psychological load [30-32].

In summary, mental load significantly affects the driver's environmental perception and driving concentration. In China, more research is needed on the driver's psychological load. For example, the team of Harbin Institute of Technology studied the relationship between the drivers physiological characteristics and driving behavior [33]. The team of Jilin University [34, 35], Sun Yat-sen University [36] and Tongji University [37] analyzed the relationship between fatigue and driving risk based on the drivers' physiological features.

From the above literature review, researchers have carried out research on the mental load characteristics of drivers. However, for research convenience, the scenario is limited to the daytime. Some studies have proposed the driver's adaptation ability in nighttime, but the driver's physiological characteristics lack intensive study in the nighttime scenario. In addition, traffic accidents in China occur frequently in the nighttime, currently. Thereby, how to combine the driver's task (primary and secondary driver's task) and improve safety in the nighttime has become an urgent issue to be addressed.

1.3. Contributions. The main contribution of this article is to study driving behavior in the nighttime scenario. It is based on 4 types of driving experiments on a driving simulator: a single driving task experiment and a master-slave driving task experiment, respectively, in nighttime and daytime. By analyzing the physiological data such as EEG, ECG, respiration, and skin electricity, and evaluating the mental load of drivers, this article reveals the variation law of drivers' physiological parameters based on statistics in the nighttime and the daytime scenario. The results can be used for road safety evaluation in the night scenario.

The remainder of the paper is organized as follows. Section 2 briefs surveys influencing factors of driver cognitive status and physiological indicators of mental workload. Section 3 presents driving simulator experiment method. The Section 4 provides driving simulator experiment results and analysis.
Section 5 is nonparametric test of driver's psychological load and actual road test. Section 6 has conclusions and related future work. The framework of this paper is illustrated in Figure 1.

\section{Influencing Factors of Driver Cognitive Status and Physiological Indicators of Mental Workload}

2.1. Factors Affecting Driver's Mental Load. The cognitive process of drivers is divided into 3 stages - the perception stage, the decision-making stage, and the operational stage.

The driver obtains outside driving environment information through sensory organs such as sight, hearing, smell, and touch during driving. Then, through the brain nerve to judge and process the information related to it, the driving behavior decision is made. Finally, the behavioral decision making for driving is performed. The mental workload refers to an additional work task that the driver needs to handle when the driver operates the vehicle, the traffic environment around it for the road restriction conditions, and the information processing capabilities on the brain that occur with frequency. This ability produces different effects and different mental stresses.

Currently when various types of ADAS continue to develop, drivers will generate higher mental workloads, especially information mental loads, and their mental load directly affects driving behavior and vehicle driving conditions. Besides, continuous driving under higher mental load is likely to cause driver judgment, decision or manipulation error, and affect road traffic safety. The main factors affecting the driver's mental load are summarized as follows.

(1) Individual characteristics affect the driver's mental load, including age, gender, education, income, travel experience, and so on. Besides, stable individual characteristics include these effects, which are about the emotional load on the driver's mental load, such as cognitive ability and cognitive style. Research result shows that different individual characteristics respond differently to the same type on outside driving or in-car driving information. However, the influence about this individual characteristic on the driver's mental load is relatively fixed. In different driving tasks, the driver's cognitive ability and cognitive style are influenced by themselves, the environment and the interaction between the two. Therefore, cognitive ability and cognitive style are in a changing state for the individual driver. And both will directly affect the driver's judgment and decision [38].

(2) The driving information search, processing and decision-making task characteristics affect the driver's mental load in three aspects: information presentation, release method, and driving environment. Based on published way information, such as color, text, symbols, and different driving environments such as weather, traffic conditions, and the outside environment, research scholars have conducted research on 


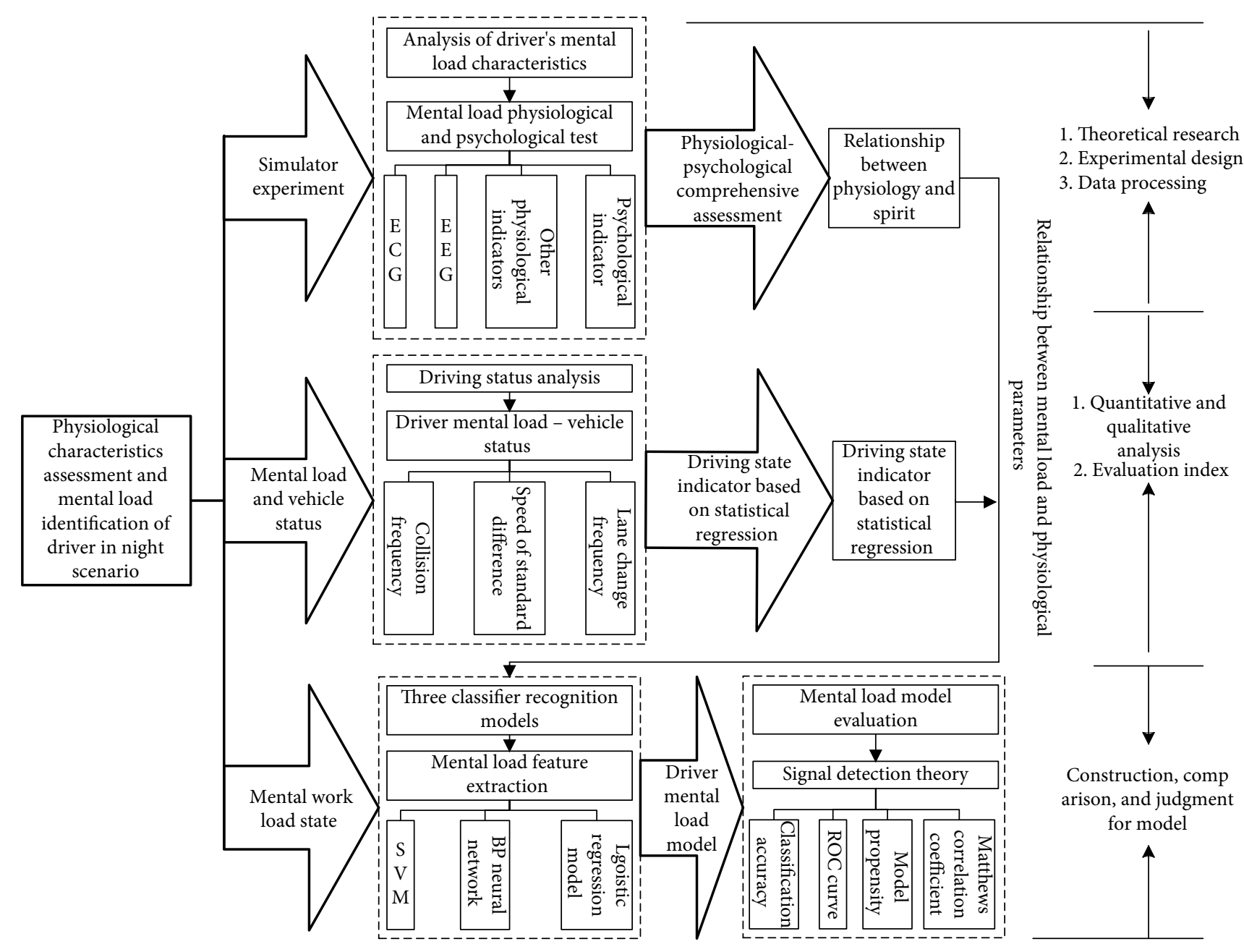

FIGURE 1: The framework of the paper.

driver's mental load in various aspects [39]. Because different information sources have differences in information release features and driver information schemas, the task complexity about information search and processing is different. Besides, different information sources will have influence on different degrees on mental workload. In addition, the road driving environment is the main influencing factor on the driver's visual and psychological load. The environmental complexity determines the intensity on the driver's mental load. Vehicle speed is an important feature in road driving environment. Many scholars have carried out research on the driver's mental load based on the relationship between vehicle speed, the driver's reaction time and operation time. Some studies have shown that higher vehicle speeds will place higher demands on the driver's response time and operating time. In the process of information search and decision, the driver will bear greater mental load [40].

2.2. Physiological Index on Driver's Mental Load. Yi-Ching Lee proposed multiple resource-related theories in his research.
They proposed that the interference generated by the two mission resources occupying two channels is much smaller than the interference generated by the same one channel [41]. According to this theory, research scholars believe that driving operations, information search, reaction decision-making, and other information processing activities in driving tasks are processes in which multiple resources share the same neural channel. With the increase in various information processing tasks, the driver's information processing ability is weakened. And the perception and driving ability in the driving environment and the surrounding area will also be weakened. Besides, this weakening is also reflected in changes in the driver's physiological indexes. Therefore, in the driving activities, human physiological parameters can reflect the psychological and physiological state objectively and accurately.

The physiological measurement sensors can record the changes of drivers various physiological data in real time and continuously. They can be utilized to evaluate the mental load of the drivers in various driving scenarios. According to generation mechanisms, the parameters are divided into 2 categories: one related to the central nervous system and the anther one is related to the peripheral nervous system. The 


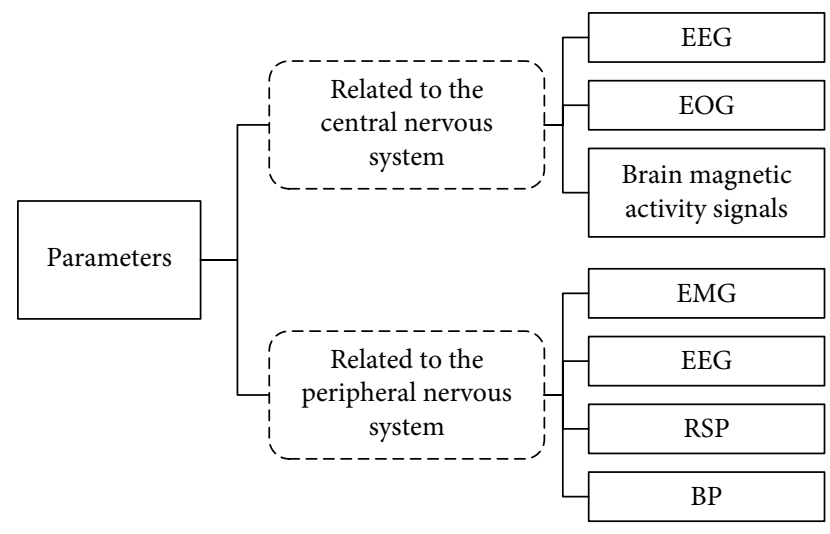

FIGURE 2: Evaluation parameters of mental load of drivers.

former covers EEG, EOG, and brain magnetic activity signals. And the latter includes EMG, ECG, RSP, BP, etc. (shown in Figure 2). To specifically evaluate the driving mental workload in daytime and nighttime scenarios, the article is based on ECG and EEG signals. This paper will select the following physiological index parameters for testing and research determination.

2.2.1. ECG Index. ECG index mainly reflects the activity on the heart. By the common dominance between the sympathetic and vagal nerves, the brain processes and judges the external information resources. The sympathetic nerve is mainly to raise the level of awakening of the organism. The vague nerve is mainly to cause the excited state organism to return to its lower arousal level. So the awakening biological level is closely related to the strength of the driver's mental load. Therefore, this paper extracts BPM, HRV, LF/HF, and other indicators in the ECG test as the test physiological indicators on the mental load intensity in the driver's multiple tasks.

2.2.2. EEG Index. During the driving task, the driver's psychological changes and subtle action decisions will be reflected in the brain waves. By monitoring changes in EEG indicators, the driver's psychological changes can be obtained objectively and accurately. The multi-information task in the driving process will stimulate the driver's visual and psychological, and then reflect in the brain waves.

2.2.3. EDA Index. The body's activity is enhanced by the stimulating effects on the sympathetic nerves. Then, the formed electrophysiological indicators can objectively reflect the changes in the activity on the sympathetic nervous system. It also directly reflects the stress changes that the brain produces when dealing with unexpected information. Drivers may experience different psychological reactions due to increased information load in the process of driving the vehicle.

2.2.4. RES Index. Increased respiratory rate as the intensity of mental load increases, the level of driver's wake-up brain, and mood swings during driving. If the driver's mental stress is too high, there will be an increase in respiratory rate.

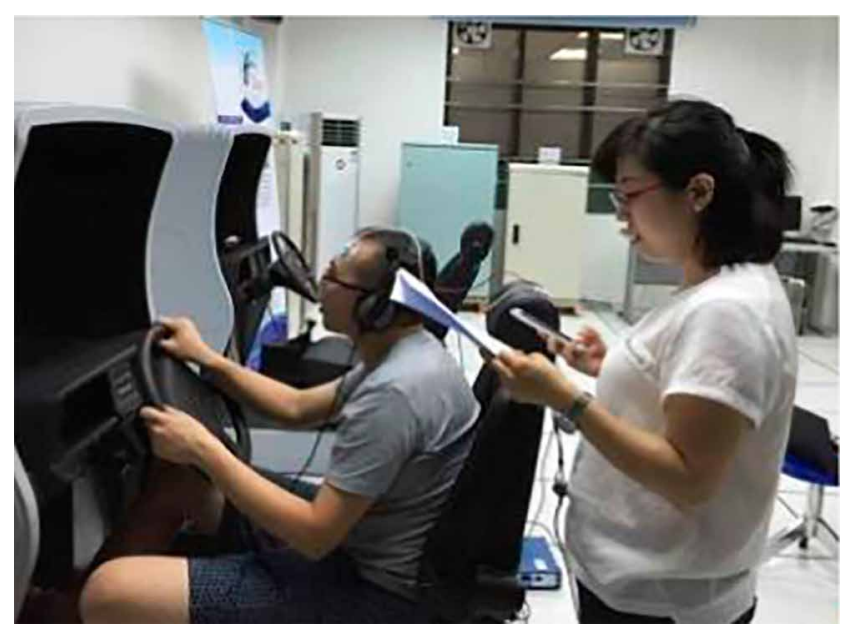

FIGURE 3: Driving simulator experiment.

\section{Driving Simulator Experiment Method}

3.1. Driving Simulator Experiment Design. Many pilot traffic behavior experiments are carried out in the driving simulator. The driving simulator can simulate different driving environments. And compared with the external road environment, the interference to the test driver's physiological instruments is smaller, and the measured human physiological data relatively more accurate. The testing experimental time is mainly based on the experimental research method on mental load in human factors engineering. Then, human subjective evaluation test method and physiological parameter acquisition method are adopted [42]. At the same time, in order to avoid the impact on physiological fatigue caused by long-time driving experiments about the experimental results, the design time for each experiment is 30 minutes.

As shown in Figure 3, for driving simulator experiment, the experiment requires the experimenter to complete the daytime and night driving experiment under the mountain highway traffic environment on the driving simulator. Each group was tested for 30 minutes. In order to reduce fatigue driving impact on various test indexes, the driver was required to rest for about 3 hours after each experiment. After the experiment, driving operation impact in single-task or master-slave task experiment is statistically analyzed when using subjective evaluation method on questionnaire and objective indicators on the physiological tester. The master-slave driving task is used to illustrate multi-task driving behavior. Here we add the conception of "multi-task driving behavior". When the driver is running a vehicle, he performs other activities simultaneously, such as talking to the passenger, turning on the air conditioner, or listening to music. At this time, driving a vehicle is a master driving task, and the others are the slave driving tasks.

The specific experimental operation steps are shown in Table 1. Before formal experiment, the experimental subject is required to perform a single driving task exercise for a temporal period to familiarize with the driving environment. The subjects were prepared for physiological patches and data acquisition, and then entered the formal driving simulator 
TABLE 1: Driving simulator experiment and master-slave driving task design.

\begin{tabular}{lc}
\hline Experiment flow & Experimental content/Experimental time \\
\hline Basic driving tasks & Familiar with driving environment of mountain roads, Data \\
Master-slave driving task one & Acquisition of Driver's Benchmark Physiology $/ 30$ min \\
Master-slave driving task two & Collection of physiological data from master-slave tasks $/ 8$ min \\
Task of test recovery & Collection of physiological data from master-slave tasks $/ 8$ min \\
\hline
\end{tabular}

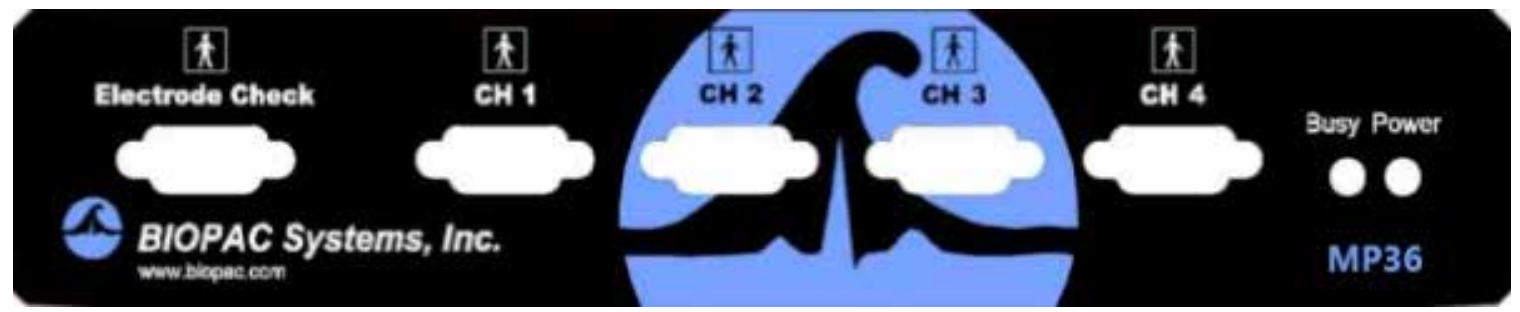

FIGURE 4: BIOPAC physiological instrument panel.

experiment stage. First, a 30 minute single driving experiment was conducted. The data in this stage are used as the benchmark data for data analysis to compare and analyze driver's changes on physiological parameters in other driving experiment tasks. Subsequently, according to the experimental content, the following experiments are carried out, respectively. The first is the master-slave driving task experiment one, which is speech memory class task. And the experiment time is $8 \mathrm{~min}$. The second is the master-slave driving task experiment two, which is simple calculation class task. And the experiment time is $8 \mathrm{~min}$. After a 5 minute recovery period, the tester parked and sat down and collected baseline physiological data indicators. Finally, the tester conducts a subjective scoring assessment on his or her own tasks. During the whole experimental process, the tester maintains uninterrupted and safe driving, and the speed is maintained at $60-70 \mathrm{~km} / \mathrm{h}$. In order to eliminate the mutual interference between the daytime or nighttime experiments, the daytime driving experiment and the night driving experiment are performed independently, and the same tester can only perform one set of experimental contents per day.

3.2. Experimental Sample. The experimental sample consists of 18 drivers with legal driving licenses aged 20-35 years old. They are healthy, have no heart disease or nervous system diseases, and they have adequate sleep and no alcohol or other drugs before the experiment.

3.3. Experiment Apparatus. For driving simulator, we set mountain highway scene in daytime and night for experiment, driving speed is $60-70 \mathrm{~km} / \mathrm{h}$, traffic flow is set as general mountain highway traffic, one-way lane, circular closed route, typical landform characteristics about mountain highway such as long longitudinal slope, bend, continuous bend, etc.

For BIOPAC MP36 named as multi-channel physiological recorder, as shown in Figure 4, it is used to monitor physiological indicators such as ECG, PEG, EEG and RES, which is as a basis for quantitative testing and evaluation on driver's mental load. Among them, the accuracy on ECG, EEG, and PEG is in the range of $10-6 \mathrm{mv}$. The breathing and heartbeat frequencies are measured in units as times percent minute. Because human physiological changes are very subtle, the selected sampling frequencies are $1000 \mathrm{~Hz}$, such as 1000 times per second while collecting experimental data. The electrocardiogram test electrode patch is located at the upper and lower body slanting sternum across the heart. EDA test electrodes stick to the left finger position, as far as possible do not interfere with driving. The breath test strap needs to be wrapped around the chest undulation, and the brain electrode patch is attached to brain forehead close to the hairline. Figure 5 shows the details of testing position to physiologic instrument.

For stopwatch, it is used to record test time. And it specifies the time of each voice task.

3.4. Experimental Content Design. When performing the experiment, the tested driver needs to continue uninterrupted driving at a speed from $60 \mathrm{~km} / \mathrm{h}$ to $70 \mathrm{~km} / \mathrm{h}$ on the set mountain highway. In the driving environment during the day and night, the driver's physiological characteristics are different, which is the key research experiment in this paper. In order to avoid the influence between different driving scenarios on the driver's physiological characteristics and mental load, the same driving scene is set in the experiment.

At the same time, when performing experiments on master-slave tasks, two voice tasks are designed as cognitive tasks in the experiment, and auditory stimulation add the speech feedback is adopted. Two voice tasks are short-term memory tasks and simple computing tasks. For short-term memory tasks, such tasks will consume drivers' short-term memory resources and occupy their cognitive channels. For example, a speech content that is repeatedly heard but each content is not repeated, subjects are required to repeat what they hear immediately, and try to maintain the correct rate of repeating content. For simple computational tasks, in order to avoid the interference on mental load caused by the problem itself, it is 


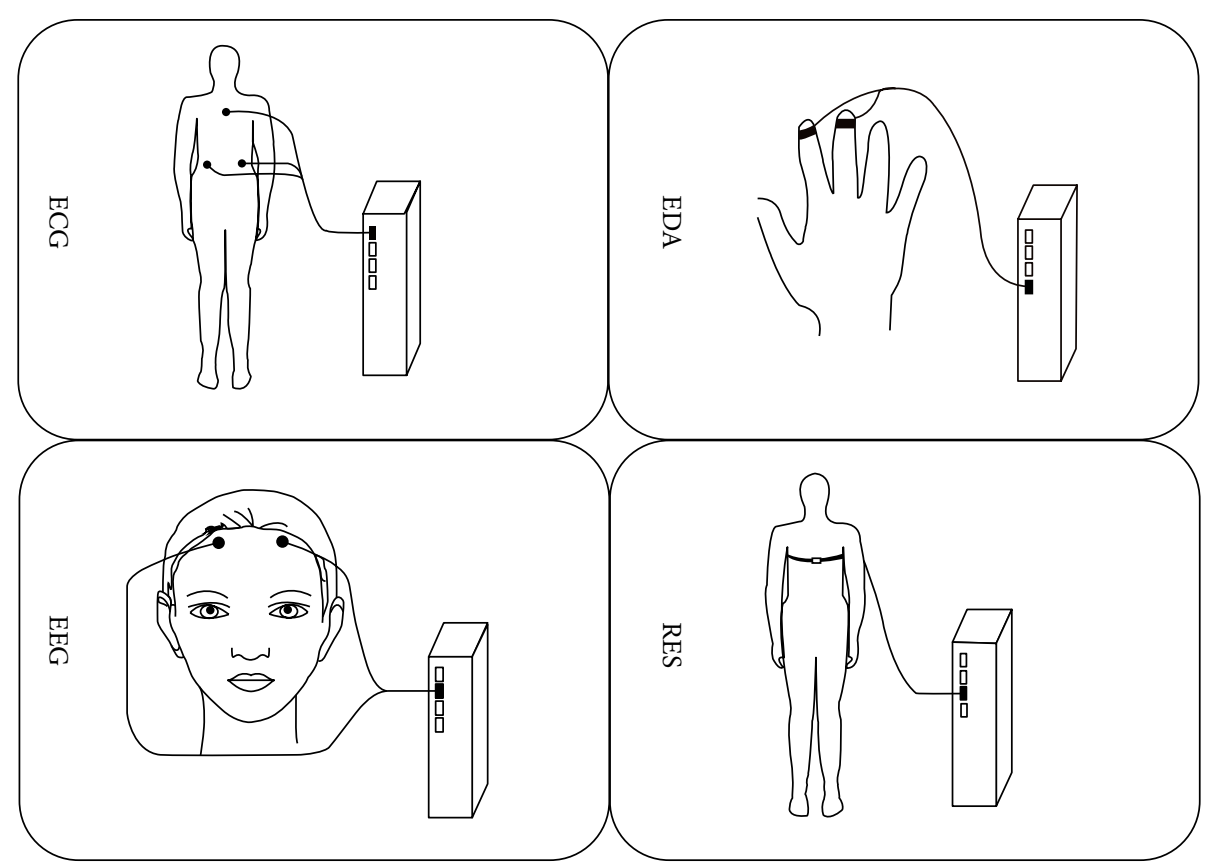

FIGURE 5: Testing position of physiologic instrument.

necessary to ensure that the difficulty coefficients are consistent and not repetitive. Besides, the subjects are asked to answer the computational questions they hear immediately, and to respond and answer in 1-2 seconds as much as possible. They are asked to answer as accurately as possible.

3.5. Measurement Methods. There are many ways to measure the driver's mental load. It can be classified into three categories: subjective evaluation measurement, behavioral performance measurement, and physiological indicator measurement [43]. The subjective evaluation measurement is a method that we measure the mental load perceived by the driver in the form of questionnaire evaluation. This method has very high effectiveness and it is easy to operate. The subjective evaluation content is based on the driver's overall experience in the completed driving task. It consists of six factors: mental load, physical demand, time demand, effort level, performance level, and frustration. The driver scores in turn from six factors from 1 to 10 points to assess the workload in the task. The behavioral performance measurement is a method that we use the driver's task performance as mental load evaluation index to derive the driver's mental load in the task. Physiological indicator measurement refers to assessing mental load by observing driver's physiological response during the task process. It can reflect the driver's mental load changes objectively and in real time.

When measuring driver's mental load, the subjective evaluation measurement method is the most main direct method to describe its subjective feelings, but it cannot describe the change process on mental load. In the evaluation process, there may be the risk of insensitivity or judgment error in its own change state judgment. And there is no standard discrimination system for the indexes. Physiological parameters are mainly ECG, EEG, EDA, RES, and so on. The evaluation method using physiological parameter index has objectivity, accuracy and dynamics, high time precision and good continuity, which can effectively correct the judgment errors on the other two evaluation methods. In order to test and verify mental load change during driving more reasonably, this paper combines physiological index test with subjective evaluation test to analyze and verify the effect between master-slave task on driver's mental load during daytime and night driving, and then we analyze and verify the effectiveness on evaluation method.

\section{Driving Simulator Experiment Results Analyzing}

The original data on ECG, EEG, RA, RF, and EDA are denoised and filtered by physiological instrument. And then physiological signals in different task stages are extracted and processed. Heart rate BPM, ECG, RR interval standard deviation, heart rate variability power spectral density, EEG four different frequency spectral density, EEG power spectral density integral value, respiratory frequency and amplitude, EDA activity level, and so on are obtained. They are used as a basis for assessing the physiological indexes at the driver's mental stress level. As shown in Figure 6, the test path starts from South China University of Technology, passing through Guangyuan Expressway, South China Expressway, Guanghe Expressway, Guangzhou-Shenzhen Expressway, etc.

When the driver performs single and master tasks in day and night, the average value about physiological indexes is extracted separately. The subjective score on the driver's mental load is based on the questionnaire evaluation method on work load methods in NASA-TLX. The subjective evaluation average scores for day and night are obtained, as shown in Tables 2 and 3. 


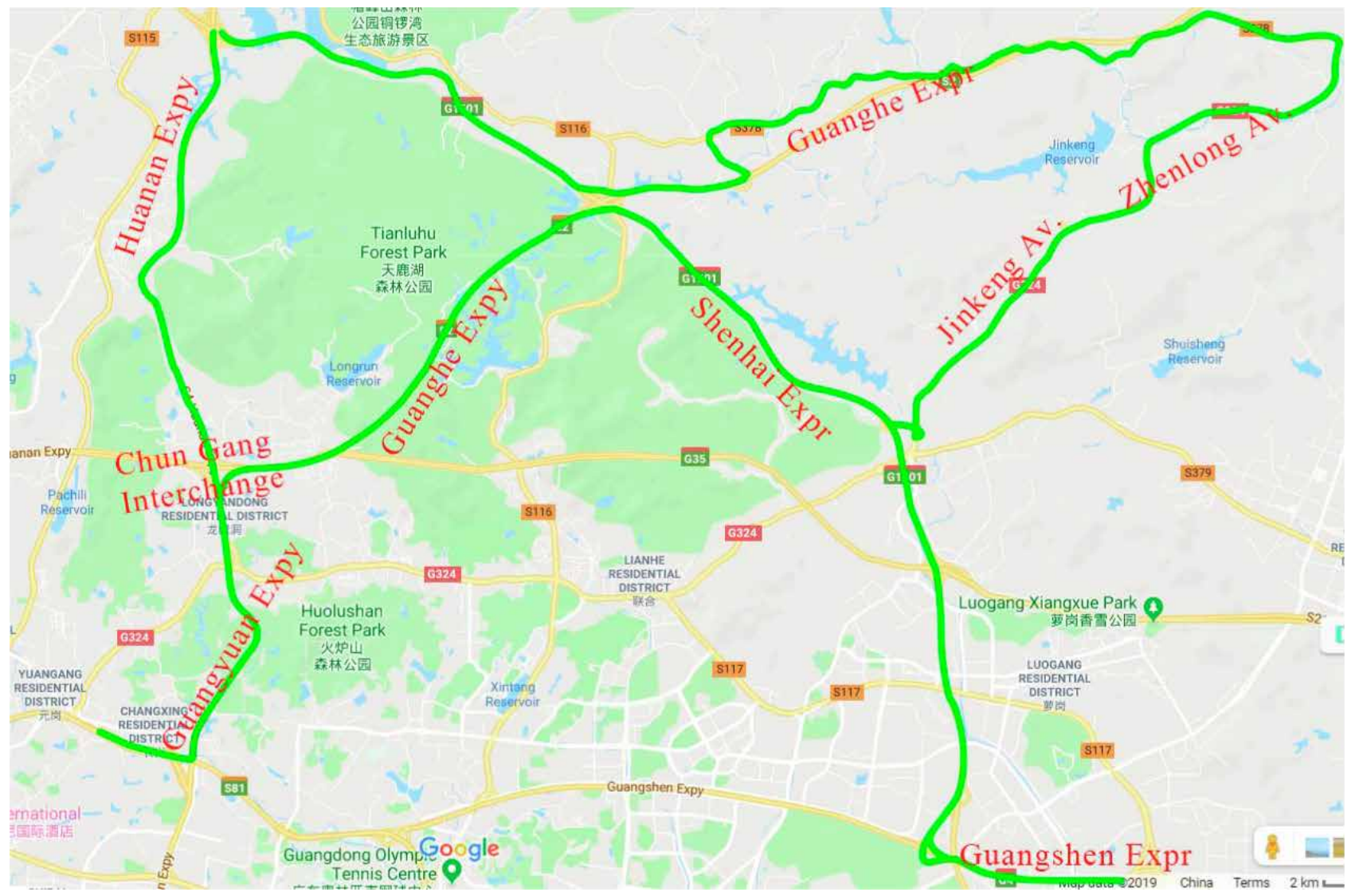

FIGURE 6: The test path for this research.

TABLE 2: Average of ECG index (day).

\begin{tabular}{lccc}
\hline Physiological index & Single driving & Master-slave task one & Master-slave task two \\
\hline MHR (mean heart rate) & 75.34 & 83.17 & 84.22 \\
RR (interval standard deviation) & 0.057 & 0.049 & 0.046 \\
LF (NU) (low frequency) & 0.769 & 0.711 & 0.558 \\
HF (NU) (high frequency) & 0.153 & 0.22 & 0.249 \\
LF/HF & 4.02 & 2.62 & 1.76 \\
\hline
\end{tabular}

TABLE 3: Average of ECG index (night).

\begin{tabular}{lccc}
\hline Physiological index & Single driving & Master-slave task one & Master-slave task two \\
\hline MHR (mean heart rate) & 74.41 & 76.58 & 77.79 \\
RR (interval standard deviation) & 0.061 & 0.046 & 0.057 \\
LF (NU) (low frequency) & 0.678 & 0.617 & 0.449 \\
HF (NU) (high frequency) & 0.197 & 0.330 & 0.425 \\
LF/HF & 2.27 & 1.68 & 1.12 \\
\hline
\end{tabular}

From the data obtained, the heart rate mean and heart rate variability indexes are similar at daytime and nighttime. The heart rate mean increases with the difficulty of the master-slave task, and the low-frequency normalized index LF (NU) in the power spectral density. The increase in task difficulty is significantly reduced. Among them, in the heart rate variability power spectral density, LF (low frequency) indicates activity intensity about sympathetic nerve, and HF (high frequency) indicates activity intensity about the vague nerve. LF/HF characterizes tension degree between two different nerves, so LF continued lowering indicates that the sympathetic excitation is reduced and the vague nerve occupies the main frequency band. Analysis on variance shows significant differences. The $\mathrm{LF} / \mathrm{HF}$ value at night is significantly lower than the daytime value, which indicates that the nondriving mission at night has more serious interference with the driver's driving process as the difficulty value increases. 


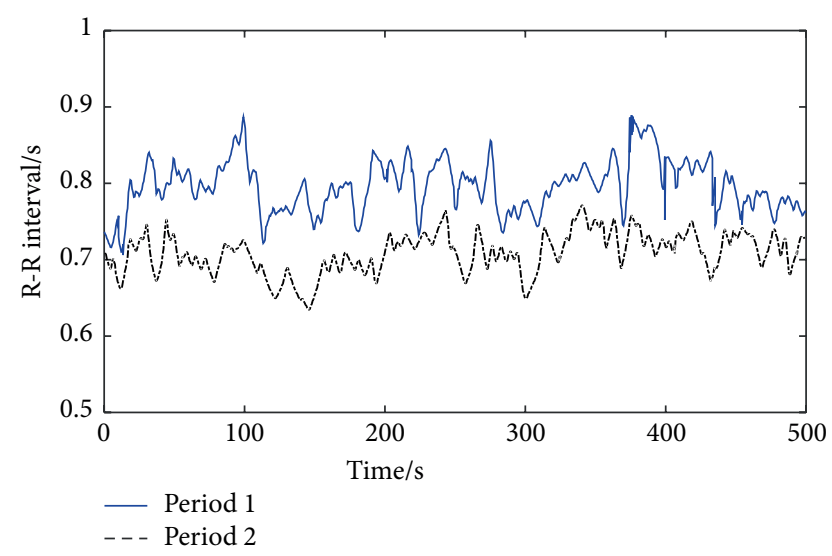

FIGURE 7: Comparison of RR interval series (single driving vs. master-slave task).

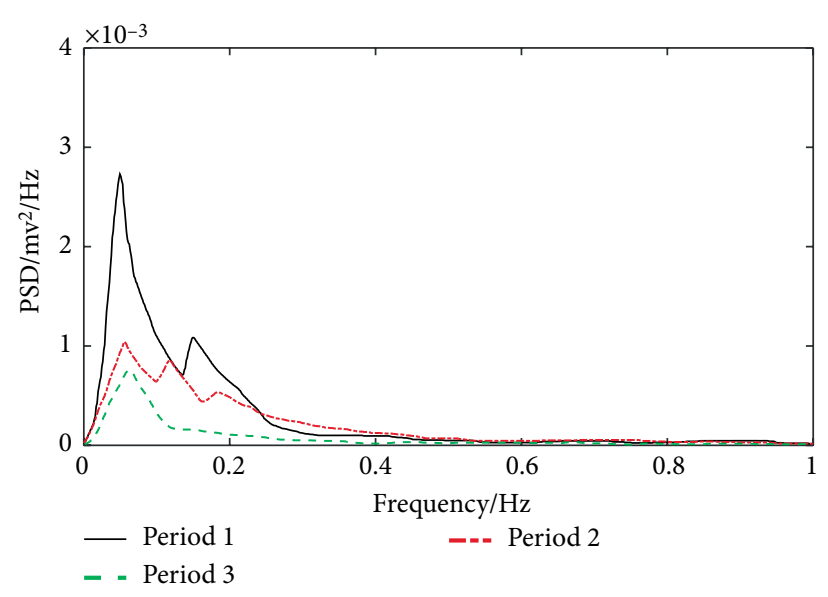

FIgURE 8: Comparison of HRV-PSD (single driving vs. master-slave task).

TABLE 4: Difference of psychological with load subjective evaluation under different driving tasks (day).

\begin{tabular}{|c|c|c|c|c|c|}
\hline Driving $\mathrm{a}-$ Driving $\mathrm{b}$ & Inspection statistics & Standard error & Statistics of standard inspection & Sig. & Significance \\
\hline Per. 1-Per. 2 & -9.448 & 5.522 & -1.713 & 0.086 & Not significant \\
\hline Per. 1-Per. 3 & -26.247 & 5.522 & -4.751 & 0.000049 & Very prominent \\
\hline Per. 2-Per. 3 & -16.788 & 5.522 & -3.044 & 0.0019 & Significant \\
\hline
\end{tabular}

TABLE 5: Difference of psychological with load subjective evaluation under different driving tasks (night).

\begin{tabular}{|c|c|c|c|c|c|}
\hline Driving $\mathrm{a}-$ Driving $\mathrm{b}$ & Inspection statistics & Standard error & Statistics of standard Inspection & Sig. & Significance \\
\hline Per. 1-Per. 2 & -16.896 & 5.522 & -3.058 & 0.0019 & Not significant \\
\hline Per. 1-Per. 3 & -30.946 & 5.522 & -5.601 & 0.000067 & Very prominent \\
\hline Per. 2-Per. 3 & -14.049 & 5.522 & -2.539 & 0.0109 & Significant \\
\hline
\end{tabular}

Figures 7 and 8 are the driver's R-R interval contrast and heart rate spectral density comparison chart, respectively, on single task and master-slave task in night environment. In Figure 7, Period 1 represents the R-R interval value at the single task phase in the nighttime environment. Period 2 represents the $\mathrm{R}-\mathrm{R}$ interval value at the master-slave task 2 . Since the R-R interval value at the master-slave task 1 phase and the single task phase does not change much during the test, Figure 7 only shows the comparison between the R-R interval values at the single task and the master-slave task 2 . In Figure 8, Period 1 represents the heart rate variability power spectral density for a single task in a nighttime environment. Period 2 represents the heart rate variability power spectral density for the master-slave task 1 . Period 3 represents the heart rate variability power for the master-slave task 2 . From overall process, it is obvious that the influence at master-slave task on the heart rate during driver's driving process varies greatly with influence on the mental load.

\section{Nonparametric Test of Driver's Psychological Load and Actual Road Test}

5.1. Nonparametric Test for Driver's Psychological Load. From the above calculation results, under the complex master-slave task conditions, the driver's subjective score has a significant increase compared to the single-task driving condition and the simple master-slave task driving situation. This difference is more significant at night. In order to determine whether these judgments are in line with statistical principles, nonparametric tests are performed on driver's subjective evaluation.

According to the characteristics for subjective evaluation data distribution, this paper chooses independent sample Kruskal-wallis test for processing. Per. 1 represents single-going driving conditions. Per. 2 represents simple master-slave mission driving conditions. Per. 3 represents complex master-slave mission driving. Under different task driving conditions, the subjective evaluation results on psychological load are shown in Tables 4 and 5.

Through the statistical numerical calculation results, there are significant differences in the psychological evaluation on mental load under three different mission driving conditions. In particular, the difficulty level at the master-slave task has a significant impact on its outcome. Among them, under the same master-slave task difficulty, the night driving stage has a more obvious impact on the driver's mental load level. This scoring result indicates that during driver's driving process, other nondriving tasks have a certain degree of adverse effect on the driver's mental load. This adverse effect is more obvious at night. 

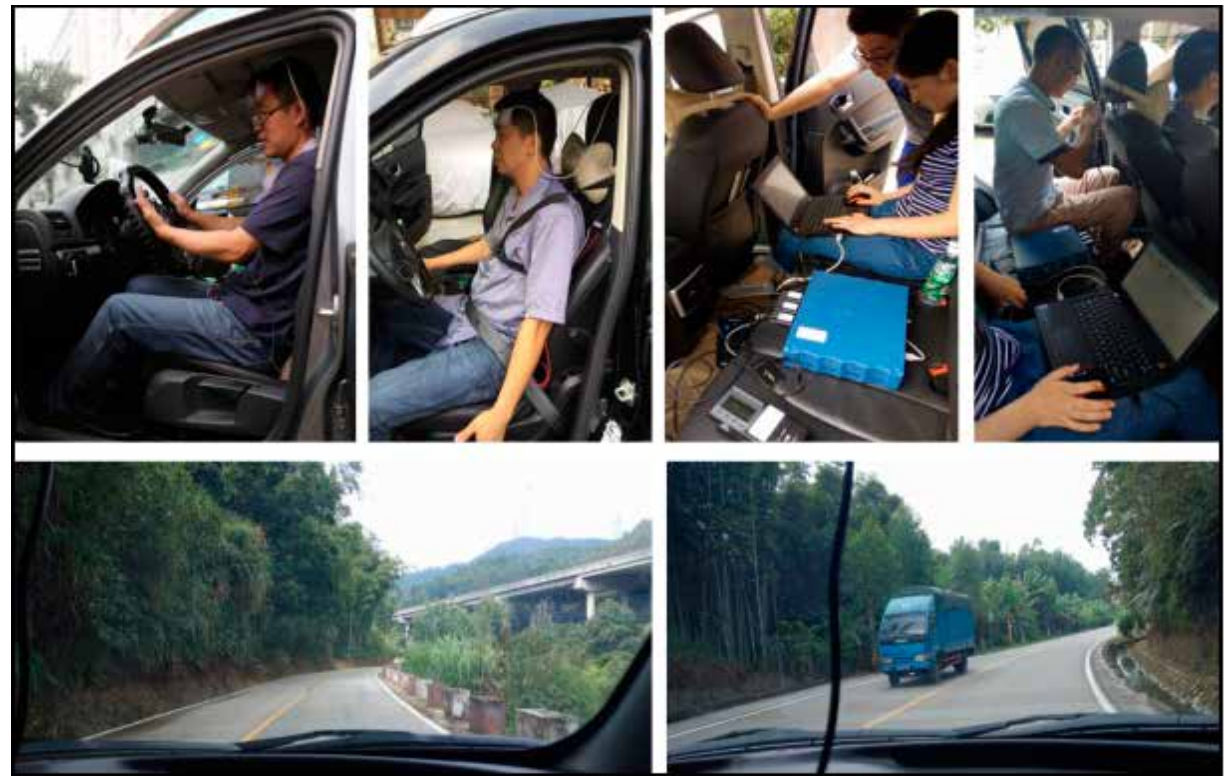

FIGURE 9: Specific experimental situation and device placement.

TABLE 6: Mean heart rate comparison of parameter results (actual road vs. driving simulator, night).

\begin{tabular}{lccc}
\hline Mean heart rate & Single driving & Simple master-slave task & Complex master-slave task \\
\hline Driving simulator & 74.39 & 76.60 & 77.82 \\
Actual road & 75.27 & 77.37 & 79.99 \\
\hline
\end{tabular}

TABLE 7: Comparison of night power spectral density LF/HF parameter results (actual road vs. driving simulator).

\begin{tabular}{lccc}
\hline Power spectral density LF/HF & Single driving & Simple master-slave task & Complex master-slave task \\
\hline Driving simulator & 2.28 & 1.69 & 1.13 \\
Actual road & 2.13 & 1.55 & 1.04 \\
\hline
\end{tabular}

5.2. Actual Road Comparison Experiment. In order to verify the authenticity on the relevant data changes in a simulated pilot experiment, the experiment designing the actual road was added on the basis of the simulated driving experiment. The experiment uses vehicles running on actual roads. At the same time, in order to make the experimental results more contrast, the participants in the actual road experiment are the drivers who have conducted simulated driving experiments. Figure 9 shows the specific experimental situation and device placement. The experiment not only selects typical mountain road sections similar to the driving simulator experiment, but also tries to balance the various road types and micro hills, hills and heavy hills on the mountain roads.

According to the experimental data analysis about the driving simulator, MHR, LF/HF, $\beta, \beta / \alpha$, and NASA-TLX in actual experiment are compared and analyzed. In order to obtain a more intuitive comparison result, the data used are normalized before the comparative analysis and then compared. This paper focuses on result comparison between simulated driver's driving data in the actual driving and driver's nighttime environment in the night environment.

The result comparison on MHR parameters is shown in Table 6. In the simulation experiment, for three driving conditions, the variation amplitudes are 2.21 and 1.22, respectively, while in the actual vehicle, the variation amplitudes are 2.1 and 2.62. It shows that driver is subjected to a higher level at mental stress when handling complex nondriving tasks for a real vehicle. For simulated driving, because it is indoor environment, the driver is relaxed, the load it bears is slightly lower. But the heart rate trend is increasing when trying to increase the interference at nondriving tasks.

The comparison about LF/HF parameter results is shown in Table 7. In the simulation experiment, the driving range in the three driving conditions is 0.59 and 0.56 . In actual driving, the decreasing ranges are 0.58 and 0.51 , respectively. The driving trends in the two driving environments are the same, and the amplitude changes are similar. Besides, it shows that driver is in a similar state to handle nondriving tasks in experimental environments.

\section{Discussion and Conclusions}

In this paper, based on the driving simulation experiment, the influence on the master-slave mission on the driver's mental load and information cognition ability during daytime and nighttime driving is analyzed. The main conclusions are as follows. 
(1) Whether it is day or night, the master-slave mission has a greater impact on the driver's mental load than on a single mission. It reduces the driver's information processing ability and increases driving risk. As the difficulty on the task which is nondriving related tasks increases and the time continues, the driver obviously feels that the subjective pressure increases, and the driving activity has an adverse effect on traffic safety.

(2) The subjective scores on the driver's mental workload questionnaire are basically consistent with the results about multi-index physiological characteristics test. The transient characteristics on physiological indexes can not only measure driver's subtle physiological changes in the subtle, but also measure the trend on mental load. This point indicates that the physiological index test is an effective test method for evaluating the driver's mental load. In the following study, an eye movement physiology index closer to the information receiving channel will be introduced as an effective evaluation method for judging mental load. It provides a more objective and feasible evaluation method for studying driver behavior.

(3) Compared to daytime, the night road environment requires a higher visual and information reception capability for the driver. Therefore, the driver's heart rate and brain electrophysiological indexes are more obvious during the day, especially in the final stage on the master-slave driving task. Drivers are more annoyed and uneasy, which can affect driving safety at night, while requiring drivers to have higher driving skills and driving risk control.

Based on research results in this paper, for drivers driving at night, it is suggested that relevant departments should consider further strengthening scientific management and adjustment of relevant regulations, which aims to minimize the risk of road traffic accidents. In the future work, we will consider increase the number on experimental samples and the difference in driver's driving experience for comparative experiments, which can verify the rationality and universality about research conclusions. Besides, we will consider the experiment under Cooperative Vehicle-Infrastructure Systems [44-47].

\section{Data Availability}

The data used to support the findings of this study are available from the corresponding author upon request.

\section{Conflicts of Interest}

The authors declare that there are no conflicts of interests regarding the publication of this article.

\section{Acknowledgments}

This work was partially supported by National Natural Science Foundation of China (51808151), and the Fundamental Research Funds for Guangdong Communication Polytechnic (20181014).

\section{References}

[1] H.-W. Wang, H.-Y. Wen, and L. I. U. Min, "Experimental evaluation of nighttime driver's physiological characteristics in driving simulator," Journal of Jilin University (Engineering and Technology Edition), vol. 47, no. 2, pp. 420-428, 2017.

[2] J. A. Veltman, "A comparative study of psychophysiological reactions during simulator and real flight," International Journal of Aviation Psychology, vol. 12, no. 1, pp. 33-48, 2002.

[3] F. Nocera, A. Couyoumdjian, and F. Ferlazzo, "Crossing the pillars of hercules: the role of spatial frames of reference in error making," The Quarterly Joural of Experimental Psychology, vol. 59, no. 1, pp. 204-221, 2006.

[4] F. Chen, H. Peng, X. Ma, J. Liang, W. Hao, and X. Pan, "Examining the safety of trucks under crosswind at bridge-tunnel section A driving simulator study," Tunnelling and Underground Space Technology, vol. 92, p. 103034, 2019.

[5] A. J. Tattersall and G. R. J. Hockey, "Level of operator control and changes in heart rate variability during simulated flight maintenance," Human Factors: The Journal of the Human Factors and Ergonomics Society, vol. 37, no. 4, pp. 682-698, 1995.

[6] W. Jian, Y. Lin-lin, Z. Bing, and D. Wei-wen, "Identification of driver individualities using Gaussian mixture model," Journal of Jilin University (Engineering and Technology Edition), vol. 1, pp. 38-43, 2015.

[7] X. Zhi, G. Hong-zhi, and Y. Hai, "Driver eye movement features on text message from traffic signs," Journal of Beijing University of Technology, vol. 37, no. 12, pp. 1830-1835, 2011.

[8] Y. Man-juan, W. Ling-tao, and T. Cheng-cheng, "Study of influence of foreign characters in guide signs on legibility," Journal of Highway and Transportation Research and Development, vol. 6, no. 2, pp. 91-95, 2012.

[9] G. Zhen-hai, D. Li-fei, Y. Zhao Hui, and Huili, "Assessment of driver's cognitive workload under multitask based on physiological signals," Automotive Engineering, vol. 1, pp. 33-37, 2015.

[10] Y. M. Li-li, "Influential factors analysis of drivers' mental workload with the use of vehicle navigation system," Journal of Safety and Environment, vol. 11, no. 6, pp. 202-204, 2011.

[11] S. Rubio, E. Diaz, J. Matin, and J. M. Puente, "Evaluation of subjective mental workload: a comparison of SWAT, NASATLX, and workload profile methods," Applied Psychology, vol. 53, no. 1, pp. 61-86, 2004.

[12] S. Pala, T. Schnell, N. L. Becklinger et al., "Adaptation of the cognitive avionic tool set (CATS) into automotive human machine interface design process," SAE Paper, vol. 1, p. 0594, 2011.

[13] M. A. Recarte and L. M. Nunes, "Mental workload while driving: effects on visual search, discrimination, and decision making," Journal of Experimental Psychology, vol. 9, no. 2, pp. 119-137, 2003.

[14] C. Bi, Y. Yuan, R. Zhang, Y. Xiang, Y. Wang, and J. Zhang, "A dynamic mode decomposition based edge detection method for art images," IEEE Photonics Journal, vol. 9, no. 6, pp. 1-13, 2017.

[15] N. Arbabzadeh and M. Jafari, "A data-driven approach for driving safety risk prediction using driver behavior and roadway information data," IEEE Transactions on Intelligent Transportation Systems, pp. 1-15, 2017.

[16] C. Bi, B. Fu, J. Chen et al., "Machine learning based fast multilayer liquefaction disaster assessment," World Wide WebInternet and Web Information Systems, pp. 1-16, 2019. 
[17] C. Bi, Y. Yuan, J. Zhang, Y. Shi, Y. Wang, and R. Zhang, "Dynamic mode decomposition based video shot detection," IEEE Access, vol. 6, pp. 21397-21407, 2018.

[18] F. Chen, M. Song, and X. Ma, "Investigation on the injury severity of drivers in rear-end collisions between cars using a random parameters bivariate ordered probit model," International Journal of Environmental Research and Public Health, vol. 16, no. 14, p. 2632, 2019.

[19] B. Yu, Z. Z. Yang, and B. Yao, "An improved ant colony optimization for vehicle routing problem," European Journal of Operational Research, vol. 196, no. 1, pp. 171-176, 2009.

[20] B. Yu, W. H. K. Lam, and M. L. Tam, "Bus arrival time prediction at bus stop with multiple routes," Transportation Research Part C, vol. 19, no. 6, pp. 1157-1170, 2011.

[21] D. Ma, X. Luo, S. Jin, W. Guo, and D. Wang, "Estimating maximum queue length for traffic lane groups using travel times from video-imaging data," IEEE Intelligent Transportation Systems Magazine, vol. 10, no. 3, pp. 123-134, 2018.

[22] D. Ma, X. Luo, S. Jin, D. Wang, and W. Guo, "Lane-based saturation degree estimation for signalized intersections using travel time data," IEEE Intelligent Transportation Systems Magazine, vol. 9, no. 3, pp. 136-148, 2017.

[23] B. Yao, P. Hu, X. Lu, J. Gao, and M. Zhang, "Transit network design based on travel time reliability," Transportation Research Part C, vol. 43, pp. 233-248, 2014.

[24] D. Ma, X. Luo, W. Li, S. Jin, W. Guo, and D. Wang, "Traffic demand estimation for lane groups at signal-controlled intersections using travel times from video-imaging etectors," IET Intelligent Transport Systems, vol. 11, no. 4, pp. 222-229, 2017.

[25] F. Chen, M. Song, X. Ma, and X. Zhu, "Assess the impacts of different autonomous trucks' lateral control modes on asphalt pavement performance," Transportation Research C: Emerging Technologies, vol. 103, pp. 17-29, 2019.

[26] R. H. Zhang, Z. C. He, H. W. Wang, F. You, and K. N. Li, "Study on self-tuning tyre friction control for developing main-servo loop integrated chassis control system," IEEE Access, vol. 5, pp. 6649-6660, 2017.

[27] X. J. Sun, H. Zhang, W. J. Meng, R. H. Zhang, K. L. Li, and T. Peng, "Primary resonance analysis and vibration suppression for the harmonically excited nonlinear suspension system using a pair of symmetric viscoelastic buffers," Nonlinear Dynamics, vol. 94, no. 2, pp. 1243-1265, 2018.

[28] F. You, R. Zhang, L. Guo, H. Wang, H. Wen, and J. Xu, "Trajectory planning and tracking control for autonomous lane change maneuver based on the cooperative vehicle infrastructure system," Expert Systems with Application, vol. 42, no. 14, pp. 5932-5946, 2015.

[29] F. Jie, Q. Liu, K. Liufu, Y. Deng, J. Fang, and Q. Li, “Design of bionic-bamboo thin-walled structures for energy absorption," Thin-Walled Structures, vol. 135, pp. 400-413, 2019.

[30] H. Xiong, X. Zhu, and R. Zhang, "Energy recovery strategy numerical simulation for dual axle drive pure electric vehicle based on motor loss model and big data calculation," Complexity, vol. 2018, pp. 1-14, 2018.

[31] H. Xiong, Z. Tan, R. Zhang, Z. Zong, and Z. Luo, "Numerical calculation model performance analysis for aluminum alloy mortise-and-tenon structural joints used in electric vehicles," Composites Part B: Engineering, vol. 161, pp. 77-86, 2019.

[32] Y. Zhang, Q. Liu, Z. He, Z. Zong, and J. Fang, "Dynamic impact response of aluminum honeycombs filled with expanded polypropylene foam," Composites Part B, vol. 156, pp. 17-27, 2019.

[33] W. Qi, Z. Wang, R. Tang, and L. Wang, "Driving risk detection model of deceleration zone in expressway based on generalized regression neural network," Journal of Advanced Transportation, vol. 2018, Article ID 8014385, p. 8, 2018.

[34] W. Lin-hong, L. Shi-wu, Z. Ru-bo, Y. Zhi-fa, J. Bin-kui, and Z. Xue-ping, "Impact of roadside landscape color on driver mean heart rate," Journal of Jilin University (Engineering and Technology Edition), vol. 1, pp. 74-80, 2013.

[35] L. Yang, B. Wang, R. Zhang, H. Zhou, and R. Wang, "Analysis on location accuracy for the binocular stereo vision system," IEEE Photonics Journal, vol. 10, no. 1, pp. 1-16, 2018.

[36] H. Xiong, M. Zhang, R. Zhang et al., "A new synchronous control method for dual motor electric vehicle based on cognitiveinspired and intelligent interaction," Future Generation Computer Systems, vol. 94, pp. 536-548, 2019.

[37] G. Wu, F. Chen, X. D. Pan, M. Xu, and X. Y. Zhu, "Using the visual intervention influence of pavement markings for rutting mitigation-part I: preliminary experiments and field tests," International Journal of Pavement Engineering, vol. 20, no. 6, pp. 734-746, 2019.

[38] L. Wang, Y. Wang, and Y. Bie, "Automatic estimation method for intersection saturation flow rate based on video detector data," Journal of Advanced Transportation, vol. 2018, Article ID 8353084, p. 9, 2018.

[39] L. Wang, L. Zhang, H. Li, Y. Ma, and R. Zhang, "High selective production of 5-hydroxymethylfurfural from fructose by sulfonic acid functionalized SBA-15 catalyst," Composites Part B: Engineering, vol. 156, pp. 88-94, 2019.

[40] S. Larue Grégoire, W. Christian, S. Michelle, and A. Rakotonirainy, "Validation of a driving simulator study on driver behavior at passive rail level crossings," Human Factors: The Journal of the Human Factors and Ergonomics Society, vol. 60, no. 6, pp. 743-754, 2018.

[41] Y.-C. Lee, J. D. Lee, and L. Ng Boyle, "Visual attention in driving: the effects of cognitive Load and visual disruption," Human Factor, vol. 49, no. 4, pp. 721-733, 2007.

[42] A. Nguyen, M. M. Borghese, and I. Janssen, "Pedestrian traffic safety and outdoor active play among 10-13 year olds living in a mid-sized city," Preventive Medicine Reports, vol. 10, pp. 304-309, 2018.

[43] C. Ma, W. Hao, W. Xiang, and W. Yan, "The impact of aggressive driving behavior on driver-injury severity at highway-rail grade crossings accidents," Journal of Advanced Transportation, vol. 2018, pp. 1-10, 2018.

[44] R. Oliveira, C. Montez, A. Boukerche, and M. S. Wangham, "Reliable data dissemination protocol for VANET traffic safety applications," Ad Hoc Networks, vol. 63, pp. 30-44, 2017.

[45] J. Casselgren and U. Bodin, "Reusable road condition information system for traffic safety and targeted maintenance," IET Intelligent Transport Systems, vol. 11, no. 4, pp. 230-238, 2017.

[46] L. Xin, L. Wu, and X. Yang, "Exploring the impact of social economic variables on traffic safety performance in Hong Kong: a time series analysis," Safety Science, vol. 109, pp. 67-75, 2018.

[47] P. Najaf, M. T. Isaai, M. Lavasani, and J.-C. Thill, "Evaluating traffic safety policies for developing countries based on equity considerations," Journal of Transportation Safety \& Security, vol. 9, no. sup1, pp. 178-203, 2017. 


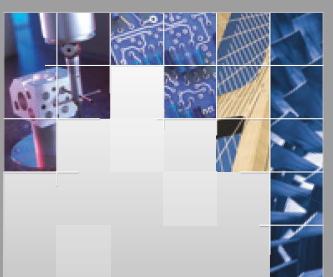

\section{Enfincering}
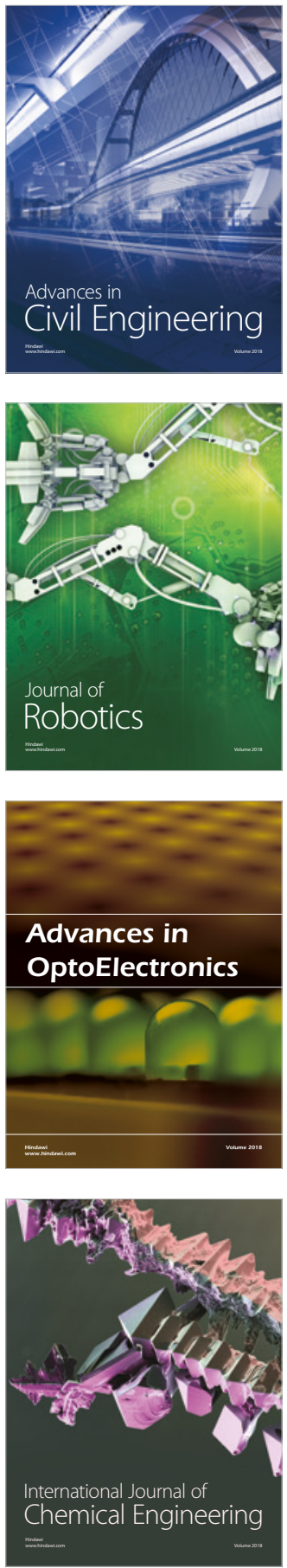

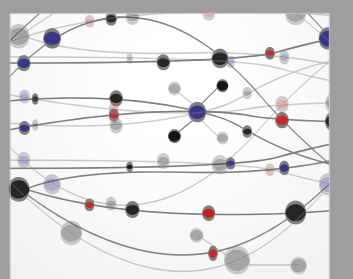

\section{Rotating \\ Machinery}

The Scientific World Journal

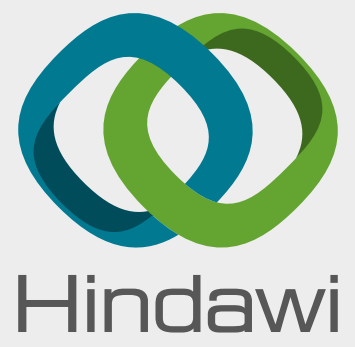

Submit your manuscripts at

www.hindawi.com
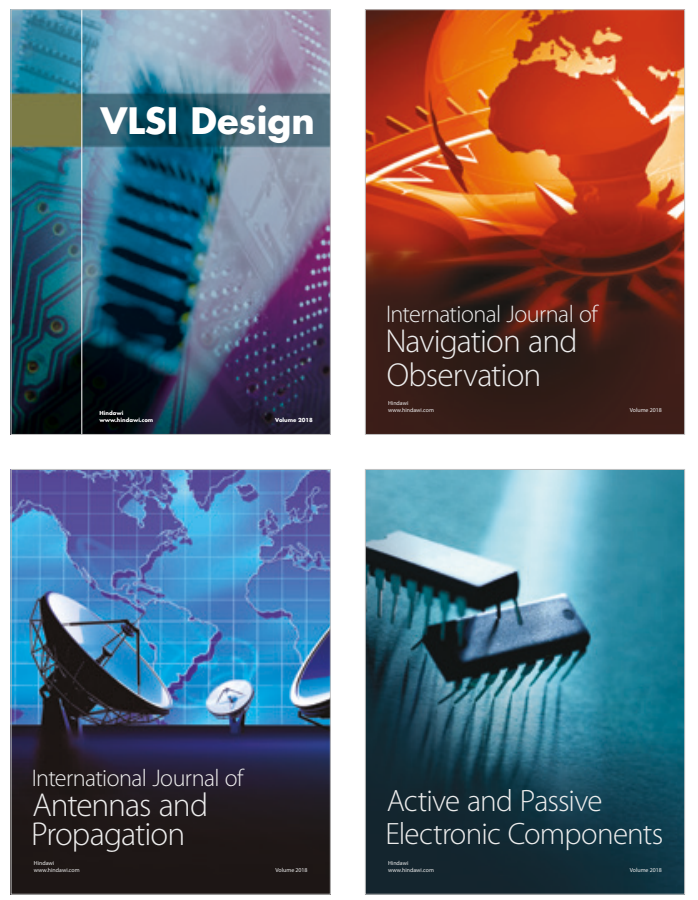
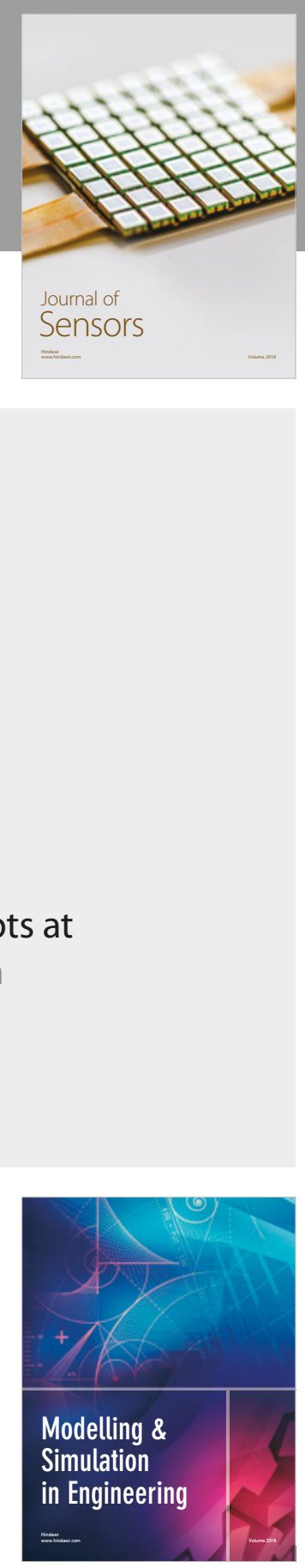

\section{Advances \\ Multimedia}
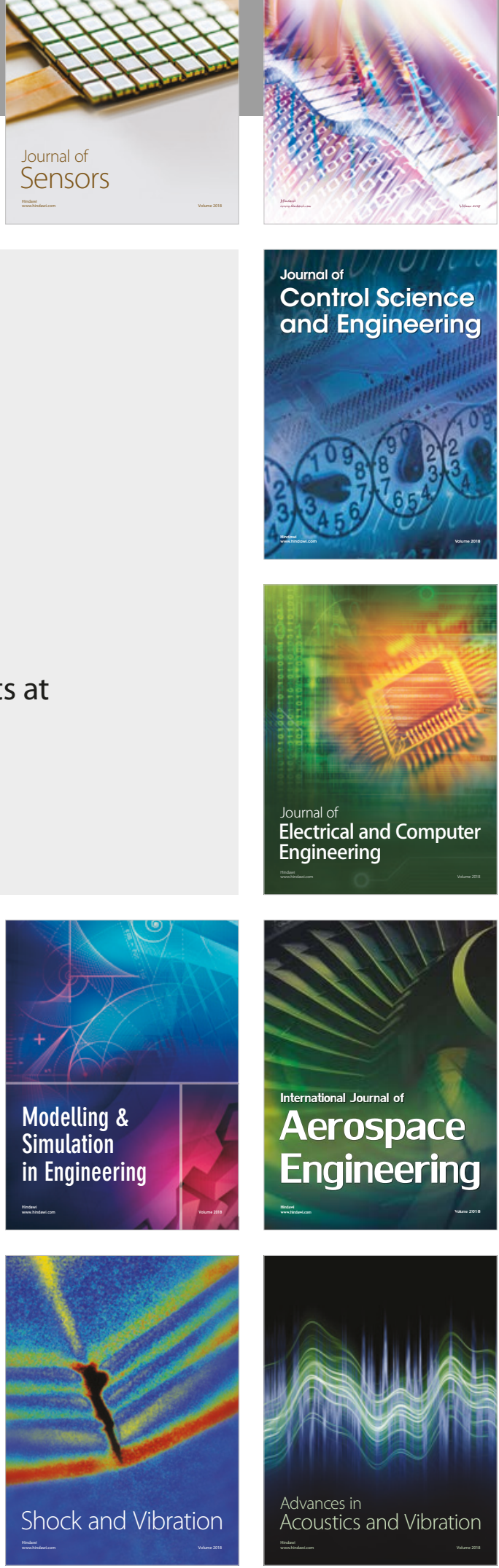